\title{
Effect of Lycra Percentages and Loop Length on the Physical and Mechanical Properties of Single Jersey Knitted Fabrics
}

\author{
Eman Eltahan \\ Textile Engineering Department, Faculty of Engineering, Alexandria University, Alexandria, Egypt \\ Correspondence should be addressed to Eman Eltahan; eman_eltahan@yahoo.com
}

Received 13 March 2016; Revised 26 June 2016; Accepted 8 July 2016

Academic Editor: Baozhong Sun

Copyright ( 2016 Eman Eltahan. This is an open access article distributed under the Creative Commons Attribution License, which permits unrestricted use, distribution, and reproduction in any medium, provided the original work is properly cited.

\begin{abstract}
Single jersey knitted fabrics are generally used to make underwear and outerwear such as T-shirts. Knit fabric can more easily deform or stretch by compressing or elongating the individual stitches that form the fabric. Cotton yarns, which are not elastomeric, do not have the ability of recovery to rearrange the stitches. As a consequence, single-knit fabrics may have permanent deformation. To improve the recovery performance of circular single-knit fabrics, it is now a common practice to knit a small amount of spandex fiber or yarn with companion cotton yarn. In this study the physical, dimensional, and mechanical properties of back plaited cotton/ spandex single jersey knitted fabrics were investigated and the results are compared with knitted fabrics made from $100 \%$ cotton and the effect of spandex percentage was also studied. It was found that as the loop length increases, the wales density was not affected and specific fabric hand and extension increased, but bursting strength and fabric recovery decreased. The presence of Lycra in single jersey knitted fabric increases of course density, fabric thickness, and knitted fabric recovery, while fabric width, fabric porosity, and extension were decreased.
\end{abstract}

\section{Introduction}

Stretch fiber, yarn, or fabric provides necessary elasticity for a garment to respond to every movement of the body and return to its original size and shape. The degree and direction of elasticity determine the end use of stretch garment. The most important property requirements for stretch garment are in the order of body comfort fit, breathability, and durability.

Haji [1] measured the physical properties of cotton/ spandex single jersey and fabric knitted from cotton alone. The statistical analysis proved that the dimensional stability and air permeability are adversely affected by the percentage of spandex. Fabric weight was improved significantly by increasing spandex ratio. He proved that fabric crease recovery was improved significantly by increasing spandex ratio. Marmarali [2] found that as the amount of spandex increased, loop length values remain nearly the same and the wale and the course spacing values decreased. Furthermore, because spandex-containing fabrics tend to be tighter, the weight and thickness of the fabrics were higher, but air permeability and spirality were lower. Kumar and Sampath [3] concluded that the wale density was not influenced but course density, area density, stitch density, and the tightness factor were all found to be inversely proportional to the stitch length value at all the relaxation states. Senthilkumar [4] found that spandex core cotton spun (SCCS) knitted fabrics were preferable for active sportswear and spandex back plaited cotton (SBPC) knitted fabrics were preferable for stretch sportswear. They also found that SCCS knitted fabric absorbs a drop of water quicker than SBPC fabric due to cotton wrapped surface in the yarn peripheral. Further, SBPC fabric has lower interyarn spaces due to higher stitch density. Prakash and Thangamani [5] found that the dimension of fabric showed considerable change during wet relaxation. The courses per inch increase from $17.65 \%$ to $70.49 \%$ and an average percentage change in courses per inch was $47.56 \%$ after wet relaxation. The wales per inch decreased from $59.57 \%$ to $31.75 \%$ and an average percentage change in wales per inch was $44.2 \%$ after wet relaxation. They concluded that the fabric shows better appearance for $2.5 \mathrm{~mm}$ loop length and $10 \%$ stretch level. Sadek et al. [6] studied the effect of Lycra percent on single jersey properties. As the Lycra extension percent reached $232 \%$ in the case of the half plating fabrics, the course density 
increased by $40 \%$, the wale density increased by $6 \%$, the thickness increased by $25 \%$, the air permeability decreased by $78 \%$, and the initial elasticity modulus decreased by $55 \%$. Also, in the case of the full plating fabrics, the course density increased by $77 \%$, the wale density increased by $14 \%$, the thickness increased by $46 \%$, the air permeability decreased by $92 \%$, and the initial elasticity modulus decreased by $69 \%$. The abrasion resistance of the full plating fabric increased by an average value of $15 \%$. Thus, the half plaiting fabrics showed better results for the course density and wale density. Zaman [7] focused his research work on dimensional properties of elastomeric single jersey knitted fabrics by feeding elastomeric yarn with different feeding speeds. He concluded that, with increasing the feeding speed, elastomeric yarn wales per inch and weight per square meter decreased. All other properties increased with increasing the feeding speed of elastomeric yarn. Cibi Vishnu et al. [8] studied the effect of heat setting and compacting on elastic properties of cotton/spandex knitted fabrics. They concluded that least time gives best elastic stretch with maximum loading whereas high elastic recovery is achieved with longer duration of heat setting. Low temperature of heat setting gave best elastic stretch and also good values of elastic recovery $\%$. But heat setting temperature has no influence on the elastic recovery of the fabric. Gokarneshan and Thangamani [9] carried out a study to find a comparative evaluation of the low stress mechanical properties of cotton/spandex and polyester/spandex blend knit. They found that extensibility increased with loop length and fine yarn fabric has more extensibility than course yarn fabric because the spandex content is more. Senthilkumar and Anbumani [10] found that fabric at gray state showed higher dynamic work recovery. After heat setting dynamic work recovery was reduced and after bleaching reduced again, this behavior was noticed in both wale and course directions. Compacting process increased the dynamic work recovery in wale direction. Effect of different stages of processing on dynamic work recovery of the fabric had significant influence. The influence of extension levels on dynamic work recovery of cotton/spandex fabrics was insignificant in case of different loop length of cotton, input tension, and spandex linear density.

\section{Materials and Methods}

The work was divided into two parts. The first part is to produce fabrics from $100 \%$ cotton with different loop lengths. In the second part, knitted fabrics were produced with different ratios of Lycra, $4.3 \%, 5 \%$, and 5.7\%, with count 22 dtex and also with different loop lengths, that is, 2.6, 2.75, 2.95, and $3.15 \mathrm{~mm}$. Cotton yarn of $40 / 1$ and spandex yarn of $22 \mathrm{dtex}$ were the main materials used to produce the knitted fabrics on a single jersey knitting machine. The normal industrial parameters were used during the process of heat setting of the cotton spandex fabrics. These parameters were temperature of $190^{\circ} \mathrm{C}$ and speed of $10 \mathrm{mt} / \mathrm{min}$. follows:

The specifications of single jersey knitting machine are as

(i) Machine type: SANTONI.

(ii) Machine diameter: 16 inches. (iii) Machine gauge: 24/inch.

(iv) Machine speed: 35 r.p.m.

(v) Number of feeders: 48 feeders.

The finishing processes of $100 \%$ cotton knitted fabrics were as follows:

(1) Bleaching.

(2) Alkaline medium.

(3) Rinsing.

(4) Neutralization.

(5) Softener.

(6) Squeezing + drying.

The finishing processes of cotton-Lycra knitted fabrics were as follows:

(1) Heat setting.

(2) Bleaching.

(3) Alkaline medium.

(4) Rinsing.

(5) Neutralization.

(6) Softener.

(7) Squeezing + drying.

The chemical material which was used in bleaching was $\mathrm{H}_{2} \mathrm{O}_{2}$, hydrogen peroxide (alkaline medium), and acid. To wet the fabric, an auxiliary material was used and then it was rinsed. An optical substance was also necessary to be added. Also softener was used and finally squeezing and drying the finished fabrics were done. The drying process was carried out at $120^{\circ} \mathrm{C}$ and a speed of $15 \mathrm{mt} / \mathrm{min}$. The final finishing process was carried out at $125^{\circ} \mathrm{C}$ and a speed of $20 \mathrm{mt} / \mathrm{min}$.

The properties of the produced knitted fabric were measured according to ASTM [11] on gray and bleached fabrics. The physical properties measured were wales and courses count, loop length, stitch density, fabric weight, and fabric thickness.

Porosity of knitted fabric can be defined as [ 1 - the ratio between the volume of the fibers in the cell and the volume of the unit cell of knitted fabric]. Therefore,

$$
\begin{aligned}
\text { Porosity\% } & =\left[1-\frac{\text { volume of fibers }}{\text { volume of cell }}\right] \times 100, \\
\text { Volume of the fibers } & =\frac{\text { weight of the yarn in the cell }}{\text { fiber density }}, \\
\text { Weight of yarn } & =L \times \frac{\text { tex }}{10^{6}},
\end{aligned}
$$

where $L$ is the loop length in $\mathrm{mm}$ and tex $/ 10^{6}$ is the weight of yarn per mm:

$$
\text { Volume of cell }=\frac{t}{c \times w} \text {, }
$$


where $(t)$ is the fabric thickness, which is $2 d$ for single jersey; that is, $t=2 d, d=0.044 \sqrt{\mathrm{tex}}$, in $\mathrm{mm}$ :

$$
\begin{aligned}
& \text { Porosity\% }=\left[1-\frac{c \times w \times L \times \text { tex }}{\rho \times 10^{6} \times 2 \times 0.044 \sqrt{\mathrm{tex}}}\right] \times 100 \%, \\
& \text { Porosity\% }=\left[1-\left(\frac{c \times w \times L \sqrt{\mathrm{tex}}}{\rho \times 88 \times 10^{3}}\right)\right] \times 100 \%,
\end{aligned}
$$

where $c=$ courses $/ \mathrm{mm}, w=$ wales $/ \mathrm{mm}, L$ is loop length in $\mathrm{mm}$, and $\rho$ is fiber density in $\mathrm{gm} / \mathrm{mm}^{3}$.

To simplify the above equation, the volume of the yarn can be calculated from another way as follows:

$$
\begin{aligned}
\text { Volume of the yarn }= & \text { loop length } \\
& \times \text { cross section area. }
\end{aligned}
$$

If the yarn is assumed theoretically to be circular in cross section, then

$$
\text { Volume of the yarn }=L \times \Pi \times \frac{d^{2}}{4} .
$$

Therefore, Porosity $\%=\left[1-\left(c \times w \times L \times \Pi \times d^{2}\right) / 4 t\right]$ :

$$
\text { Porosity } \%=\left[1-\left(\frac{c \times w \times L \times \Pi \times d}{8}\right)\right] \times 100 \% .
$$

Fabric settings $K_{c}, K_{w}$, and $K_{s}$ and loop shape factor are calculated according to Munden [12], while

$$
\begin{aligned}
K_{w} & =w \times L, \\
K_{c} & =c \times L, \\
K_{s} & =K_{c} \times K_{w}, \\
\text { Loop shape factor } & =\frac{K_{c}}{K_{w}} .
\end{aligned}
$$

The fabric setting is expressed by the number of wales per $\mathrm{cm}$ width of cloth and the number of courses per $\mathrm{cm}$ length of cloth.

The tested mechanical properties of knitted fabric were the bursting strength, elongation, and tensile recovery to represent the percentages of recovery and remaining extension in the fabric due to applying a certain load on the finished fabric.

\section{Results and Discussion}

3.1. Physical and Dimensional Properties. From Figure 1, the wales/cm decrease as the loop length increases whatever the percentage of Lycra in the fabric. This is because the Lycra in the yarn tends to contract the loops to each other.

From Figure 2, by adding Lycra in the knitted fabrics, the course density increases and the contraction of the fabric in the lengthwise direction was observed by increasing the percentage of Lycra. This is already very clear at different loop length where there is a reduction in course density by increasing the fed loop length.

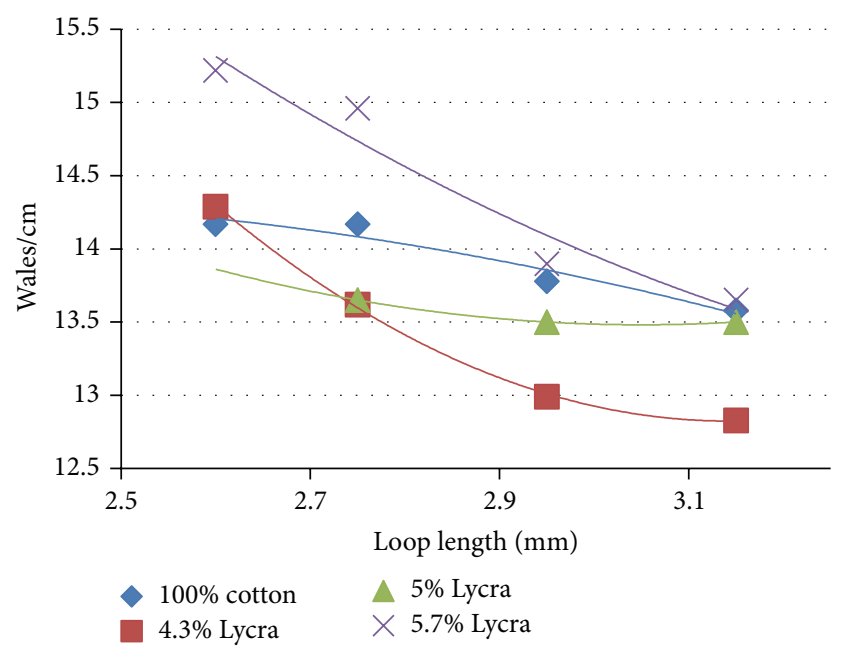

FIGURE 1: Effect of loop length on wales/cm at different percentages of Lycra.

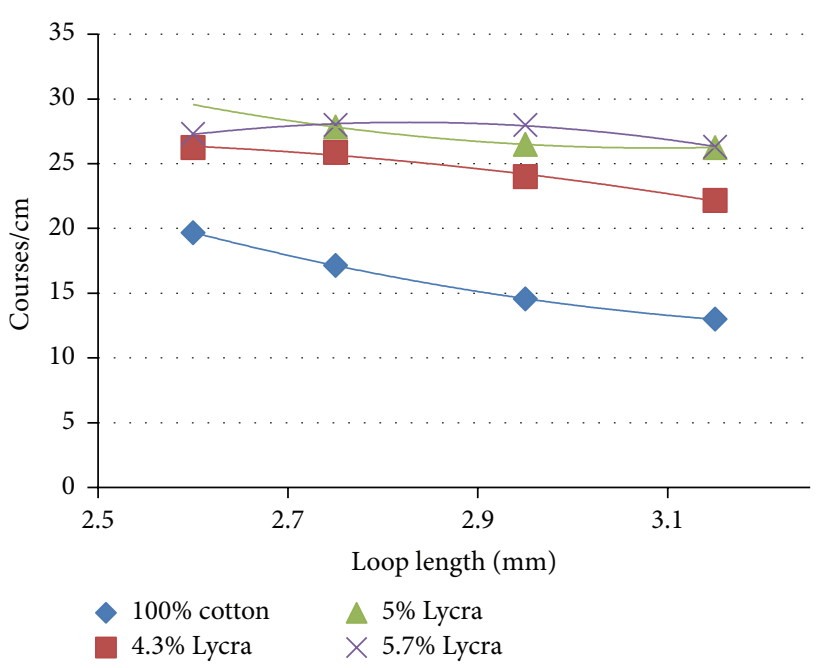

FIGURE 2: Effect of loop length on courses/cm at different percentages of Lycra.

The effect of loop length on shape factor is similar to the effect of loop length on course and wales density. As shown in Figure 3, as the loop length increases, the loop shape factor $K_{c} / K_{w}$ decreases, because when the loop length increases, the course density and wales density decrease.

It is obvious from Figure 4 that the weight of the fabric decreases as loop length increases in case of $100 \%$ cotton. This is because the increase in loop length gives an increase in the length of the loop legs and therefore the courses become far away from each other and consequently the course density decreases. But in the presence of the Lycra as the loop length increases the fabric weight slightly increases. This means that the effect of Lycra in the knitted fabric is greater than the effect of the increase in loop length which leads to compacting the fabric together. This case is at any percentage of Lycra yarn that is at $4.3 \%$ or $5 \%$ and $5.7 \%$. 


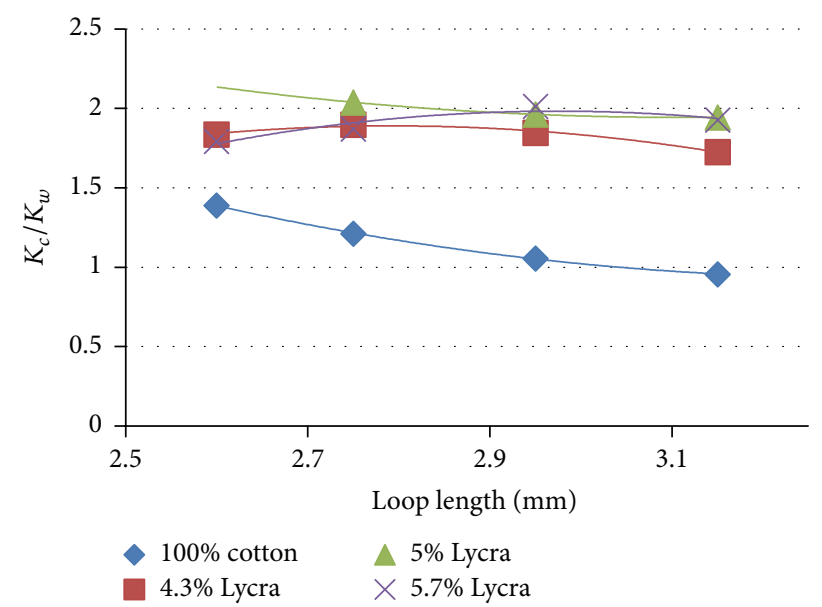

FIGURE 3: Effect of loop length on loop shape factor at different percentages of Lycra.

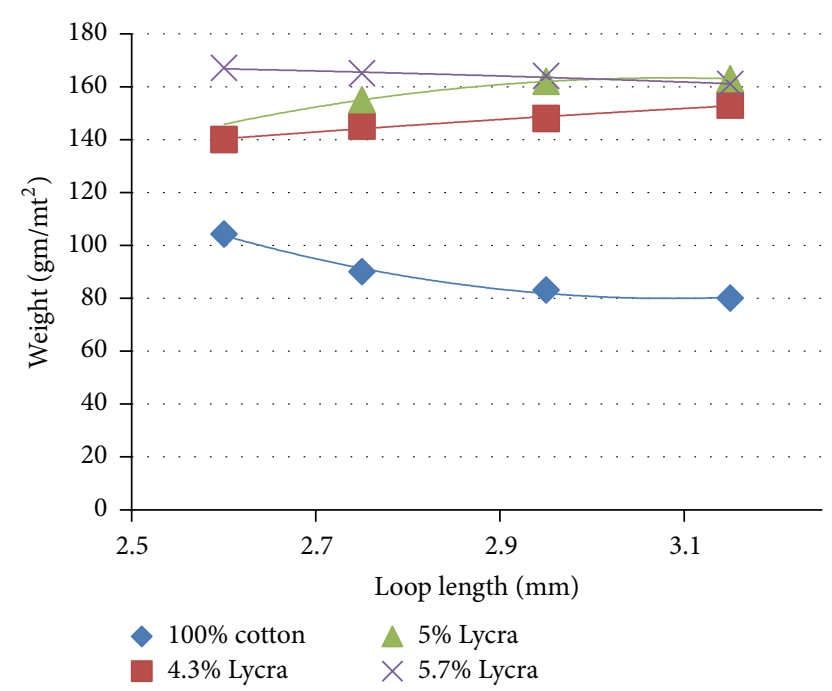

FIGURE 4: Effect of loop length on fabric weight at different percentages of Lycra.

From Figure 5, in case of $100 \%$ cotton the thickness of the fabric decreases. This is because as the loop length increases the fabric becomes more open so the mobility of the loops also increases and this leads to slipping the loops over each other when the pressing foot of the thickness tester presses the yarns which give lower thickness. The presence of Lycra tends to contract the loops to each other; therefore, the fabric thickness increases and as mentioned before the effect of the Lycra canceled the effect of the increase in loop length.

Figure 6 shows that the increase of loop length increases the fabric width. The percentage of $5.7 \%$ Lycra shows the least width of fabric. This is due to the high shrinking ability of Lycra causing the contraction in width. The $100 \%$ cotton fabric gives nearly the same width as the other fabrics with less percentage of Lycra than $5.7 \%$. This shows that the percentage of Lycra determines the effectiveness of loop length on fabric width. It is clear that the increase of loop length is more

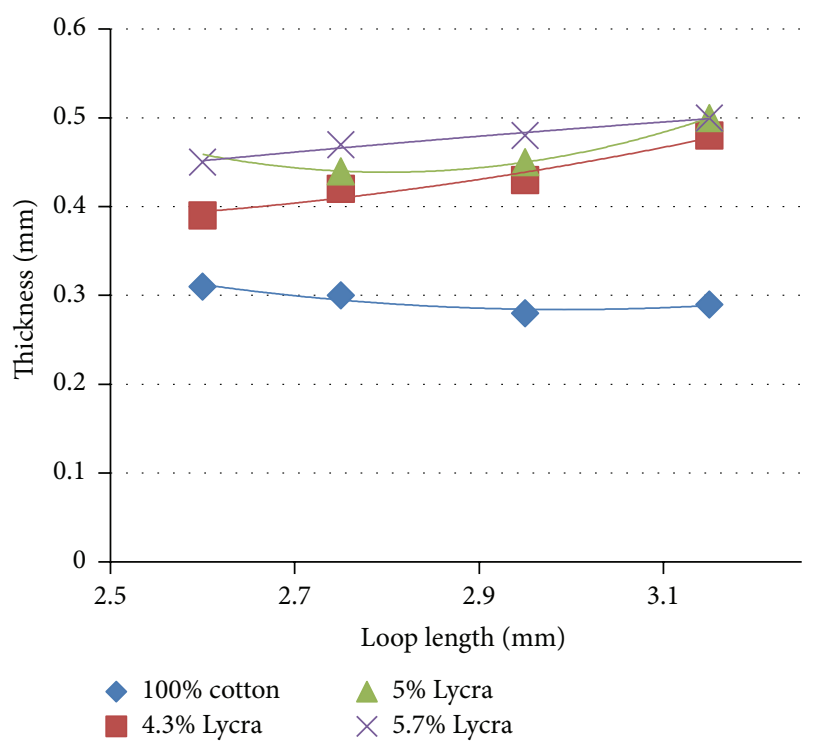

FIGURE 5: Effect of loop length on fabric thickness at different percentages of Lycra.

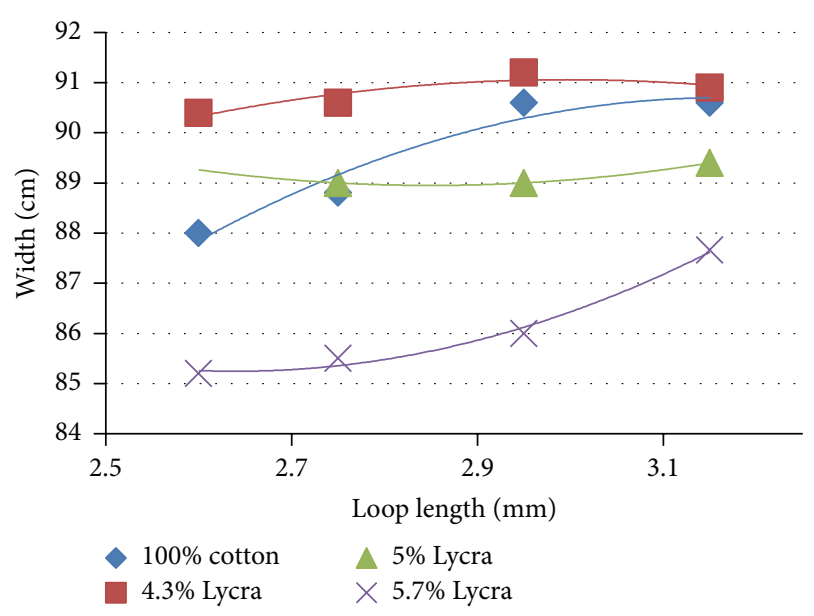

Figure 6: Effect of loop length on fabric width at different percentage of Lycra.

effective in case of $5.7 \%$ than the other fabrics with lower percent of Lycra.

Because the fabric porosity has a reflection on the air permeability, that is, as the porosity of the fabric increases the air permeability of fabric also increases, the fabric air permeability increases as the loop length increases and also as the Lycra percentage becomes lower and this is clear from Figure 7.

3.2. Mechanical Properties. It is clear from Figure 8 that the knitted fabrics made from $100 \%$ cotton show an increase in the specific hand as the loop length increases. This is because as the loop length increases the knitted fabric becomes more open and soft as compared with the small loop length. By introducing Lycra yarn in the fabric, the Lycra yarn holds the loops together and the fabric becomes less open and this 


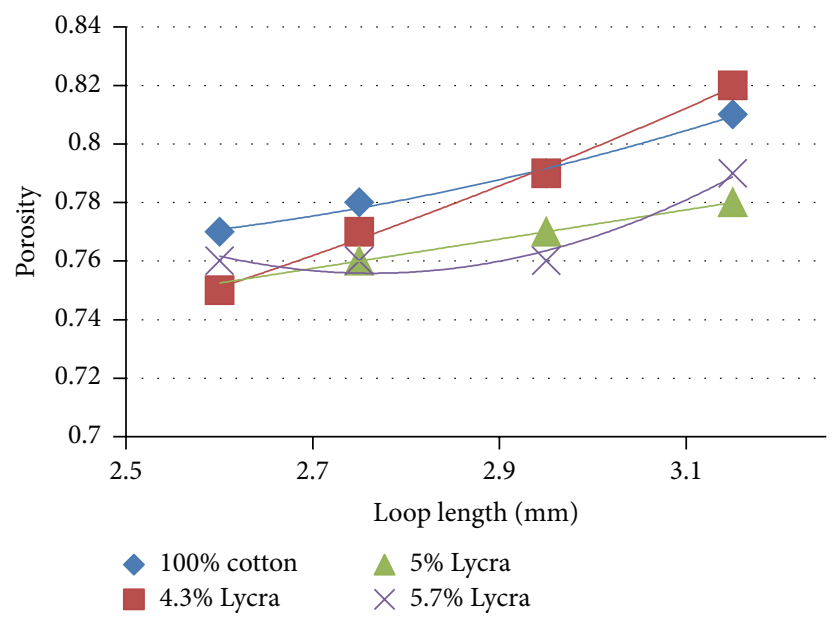

Figure 7: Effect of loop length on fabric porosity at different percentages of Lycra.

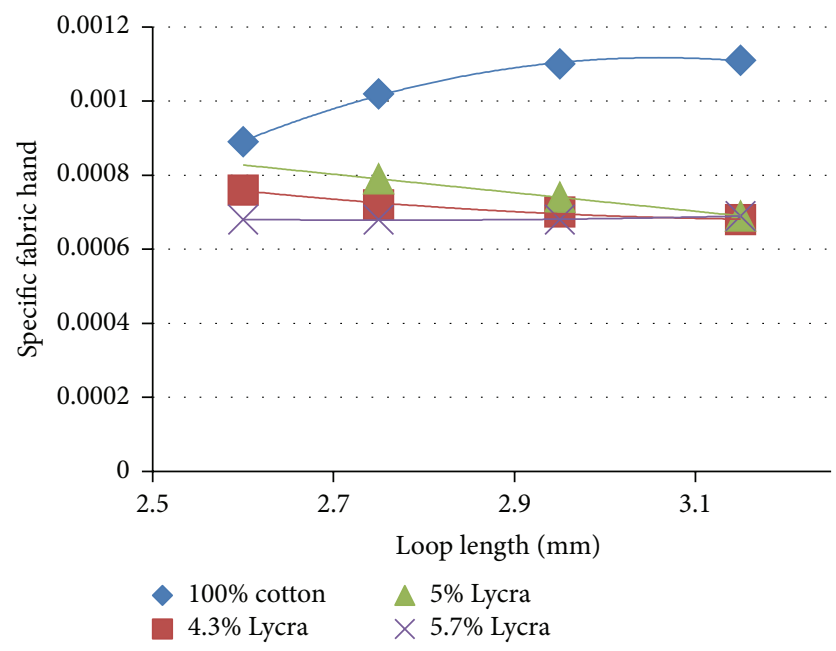

Figure 8: Effect of loop length on specific hand at different percentages of Lycra.

openness decreases by increasing the percentage of Lycra yarn. Due to the reduction in fabric openness and by inserting Lycra in the knitted fabric, the fabric specific hand decreases. It is also remarkable that the Lycra yarn can cancel the effect of loop length. It can be noticed that the fabric hand of fabrics with Lycra is lower than that of $100 \%$ cotton due to the lower bending stiffness of Lycra filaments.

It is obvious from Figure 9 that there is a reduction in bursting strength by increasing the loop length whatever the percentage of Lycra is. This is because the structure of the fabric becomes more open. In case of $100 \%$ cotton and cotton with low percentage of Lycra, the behavior of bursting strength is approximately the same as in $100 \%$ cotton knitted fabric. By increasing the percentage of Lycra to $5 \%$ and $5.7 \%$, the bursting strength is higher than in case of cotton $100 \%$ and 4.3\% Lycra. This means that the Lycra yarn causes the increase of the strength of the fabric due to the higher compactness in the fabric structure resisting the bursting force.

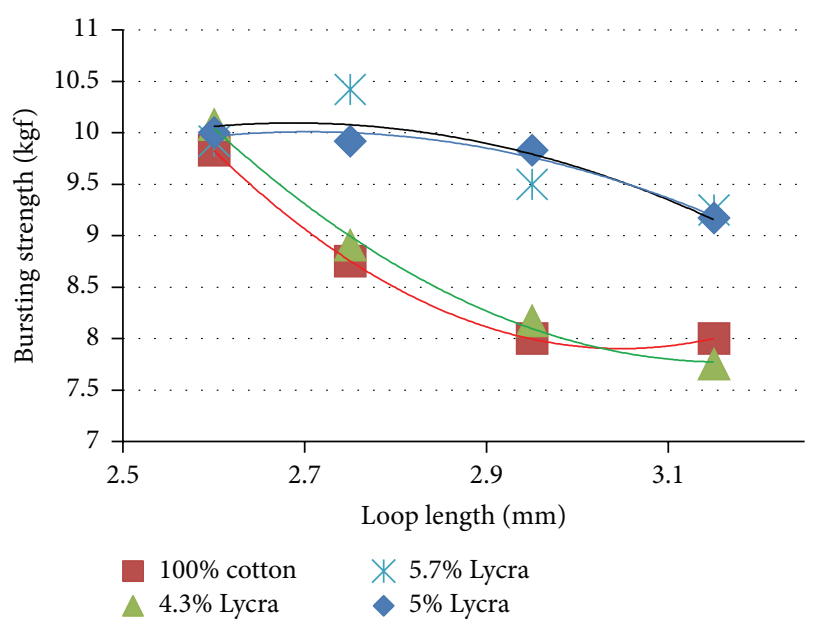

FIGURE 9: Effect of loop length on fabric bursting strength at different percentages of Lycra.

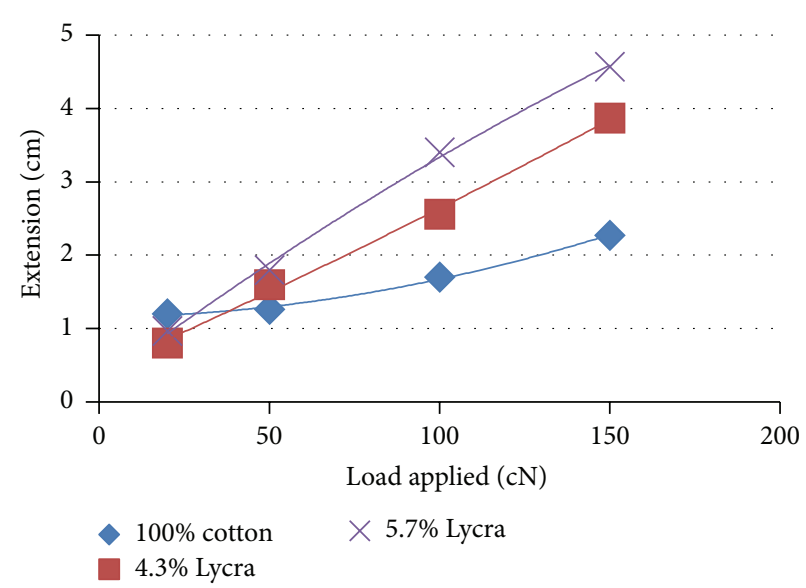

FIGURE 10: Effect of different loads in wales direction on fabric extension at loop length $=2.6 \mathrm{~mm}$.

Figure 10 shows the effect of applying different values of loads on knitted fabric extension in wales direction at loop length $2.6 \mathrm{~mm}$ which is recommended in practice. By increasing the load applied, the knitted fabric extension increases. The rate of increase in the fabric extension increases as the percentage of Lycra increases. The lowest increase in the knitted fabric in wales direction is in case of $100 \%$ cotton followed by $4.3 \%$ Lycra and then $5.7 \%$. This is because the cotton/Lycra yarn has a higher percentage of extension in comparison to the $100 \%$ cotton yarn which consequently helps to give the knitted fabric more extensibility.

By increasing the loads in the course direction, the knitted fabric extension also increases. The rate of increase of extension in the case of applying the load in course direction (Figure 11) is slightly higher than the rate of increase of extension in wales direction. This is due to the nature of the formation of the knitted loop. But the effect of the Lycra yarn in case of extension in course direction is less than in case of extension in wales direction. 


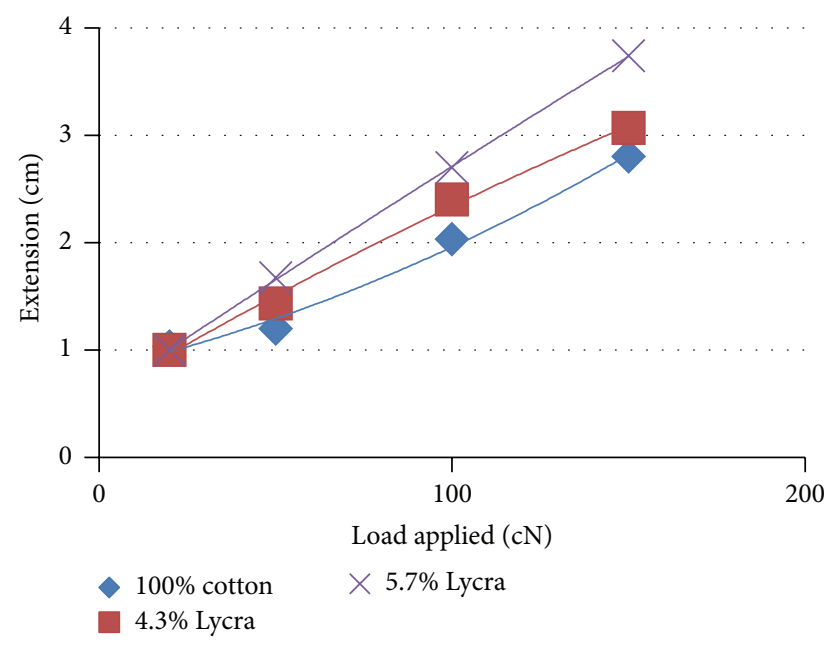

FIGURE 11: Effect of different loads on fabric extension in course direction at loop length $=2.6 \mathrm{~mm}$.

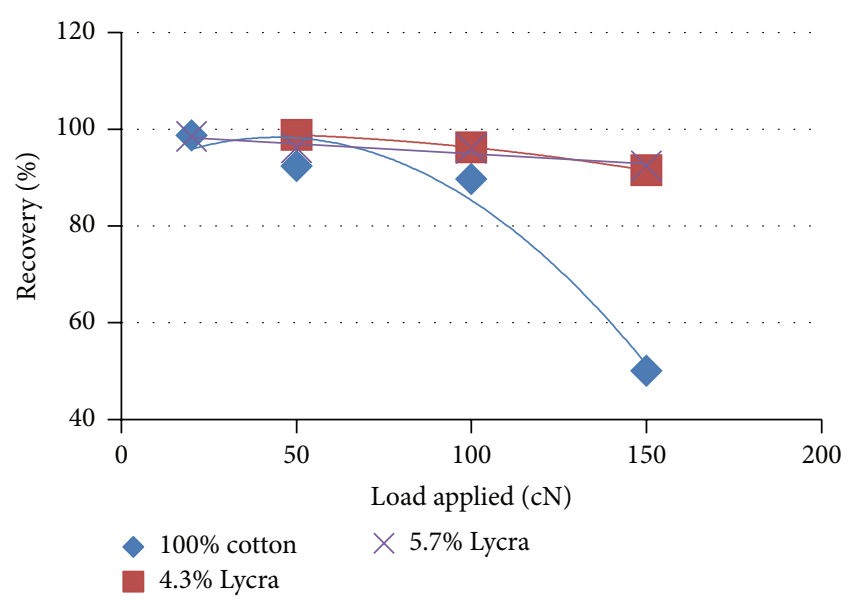

FIGURE 12: Effect of different loads on elastic fabric recovery in wales direction at loop length $=2.6 \mathrm{~mm}$.

From Figure 12, it can be noticed that as the applied load increases the percentage of elastic recovery decreases. At small load, the recovery in case of $100 \%$ cotton and in presence of Lycra yarn is the same. By increasing the load applied, the percentage of fabric recovery decreases. The lowest decrease in fabric recovery is at knitted fabric made from $100 \%$ cotton and the percentage of Lycra improves the recovery of the knitted fabric as the percentage of Lycra increases. This is due to the gain of extensibility by introducing Lycra yarn in the knitted fabric. The reason is that the fabric elastic recovery decreases by increasing the applied load when exceeding the elastic limit.

From Figure 13, it can be noticed that by increasing the load in course direction, this gave a decrease in fabric recovery. In the case of loop length $2.6 \mathrm{~mm}$ which is practically recommended, the recovery in courses and wales direction, at small load, is the same. The recovery in case of $100 \%$ cotton is less than in case of fabric with Lycra due to the higher elasticity of Lycra compared to cotton. Also, when the

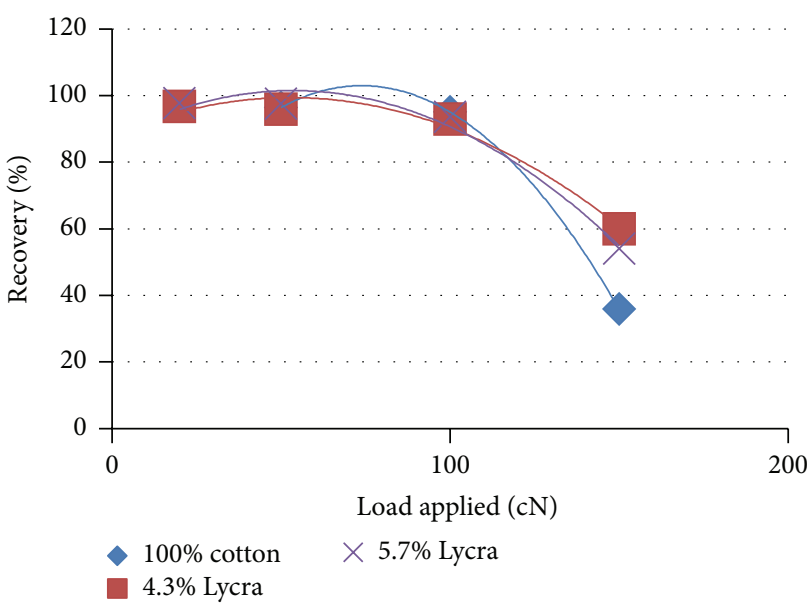

FIGURE 13: Effect of different loads on elastic fabric recovery in course direction at loop length $=2.6 \mathrm{~mm}$.

percentage of Lycra in the fabric increases, the percentage of recovery increases due to the same reason of the higher elasticity of Lycra filament.

\section{Conclusion}

From the previous results and discussion, the following can be concluded:

(1) Both loop length and percentage of Lycra in jersey knitted fabric have a great effect on knitted fabric properties.

(2) As the loop length increases, wales/unit length and courses/unit length decrease.

(3) Loop shape factor decreases as loop length increases; this is very clear in case of $100 \%$ cotton while by adding Lycra to the fabric and due to the tightness of fabric the stitch density is approximately the same.

(4) Generally, as loop length increases, the stitch density decreases and consequently weight of fabric decreases. This is in case of $100 \%$ cotton, while by adding Lycra yarn the knitted fabric becomes tight and the fabric weight increases as the percentage of Lycra increases; that is, the loop length has no significant effect on the fabric weight.

(5) Fabric thickness is not affected with loop length in case of $100 \%$ cotton, while by introducing Lycra to the knitted fabric thickness increases as the loop length increases; this increase is due to the tightness of the fabric.

(6) Fabric width increases, as the loop length increases; the increase in width in case of $100 \%$ cotton is higher than with $5.7 \%$ Lycra.

(7) Specific fabric hand in case of $100 \%$ cotton increases with the increase of loop length, while this property is approximately the same at different percentages of Lycra as the loop length increases. 
(8) Bursting strength decreases as the loop length increases, in case of $100 \%$ cotton and low percentage of Lycra. But by increasing percentage of Lycra there is a slight decrease in bursting strength as the loop length increases.

(9) The porosity of single jersey knitted fabric increases, as loop length increases; this is in all cases.

(10) Extension in wales direction of the single jersey knitted fabric increases as the load on the fabric increases; the increase in extension in case of Lycra knitted fabric is higher than $100 \%$ cotton and also the percentage of extension increases as the percentage of Lycra increases.

(11) The extension in course direction is higher than the percentage of extension in wales direction under the same load. This is in case of $100 \%$ cotton.

(12) As the load increases, the percentage of recovery decreases. The reduction in recovery in case of $100 \%$ cotton at higher loads is more than Lycra knitted fabric.

\section{Competing Interests}

The author declares that they have no competing interests.

\section{References}

[1] M. M. A. Haji, "Physical and mechanical properties of cotton/ spandex fabrics," Pakistan Textile Journal, vol. 62, no. 1, p. 52, 2013.

[2] A. B. Marmarali, "Dimensional and physical properties of cotton/spandex single jersey fabrics," Textile Research Journal, vol. 73, no. 1, pp. 11-14, 2003.

[3] V. Kumar and V. R. Sampath, "Investigation on the physical and dimensional properties of single jersey fabrics made from cotton sheath-elastomeric core spun," Fibres \& Textiles in Eastern Europe, vol. 99, no. 3, pp. 73-75, 2013.

[4] M. Senthilkumar, "Elastic knitted fabrics for tight fit sportswear," Journal of Industrial Textiles, vol. 41, no. 1, pp. 13-24, 2011.

[5] C. Prakash and K. Thangamani, "Establishing the effect of loop length on dimensional stability of single jersey knitted fabric made from cotton/lycra core spun yarn," Indian Journal of Science and Technology, vol. 3, no. 3, pp. 287-289, 2010.

[6] R. Sadek, A. M. El-Hossini, A. S. Eldeeb, and A. A. Yassen, "Effect of lycra extension percent on single jersey knitted fabric properties," Journal of Engineered Fibers and Fabrics, vol. 7, no. 2, pp. 11-16, 2012.

[7] M. Zaman, The Effect of Feeding Speeds of Elastomeric Yarn on Dimensional Properties of Single Jersey Knit Fabric, Faculty of Textile Engineering Department, International University, Dhaka, Bangladesh, 2013.

[8] C. Cibi Vishnu, L. Padmaraj, H. Sukanya, B. Suresh Kumar, N. Anbumani, and M. Senthil Kumar, Effect of Heat Setting and Compacting on Elastic Properties of Cotton/Spandex Knitted Fabrics, Department of Textile and Apparel Technology, PSG Polytechnic College, Coimbatore, India.

[9] N. Gokarneshan and K. Thangamani, "A comparative evaluation of the low stress mechanical properties cotton/spandex and polyester/spandex blend knits," International Journal of Applied Engineering and Technology, vol. 2, no. 3, pp. 23-27, 2012.

[10] M. Senthilkumar and N. Anbumani, "Effect of laundering on dynamic elastic behavior of cotton and cotton/spandex knitted fabrics," Journal of Textile and Apparel, Technology and Management, vol. 7, no. 4, 2012.

[11] https://www.astm.org/Standards/textile-standards.html.

[12] D. L. Munden, "The geometry and dimensional properties of plain-knit fabrics," Journal of the Textile Institute Transactions, vol. 50, no. 7, pp. T448-T471, 1959. 

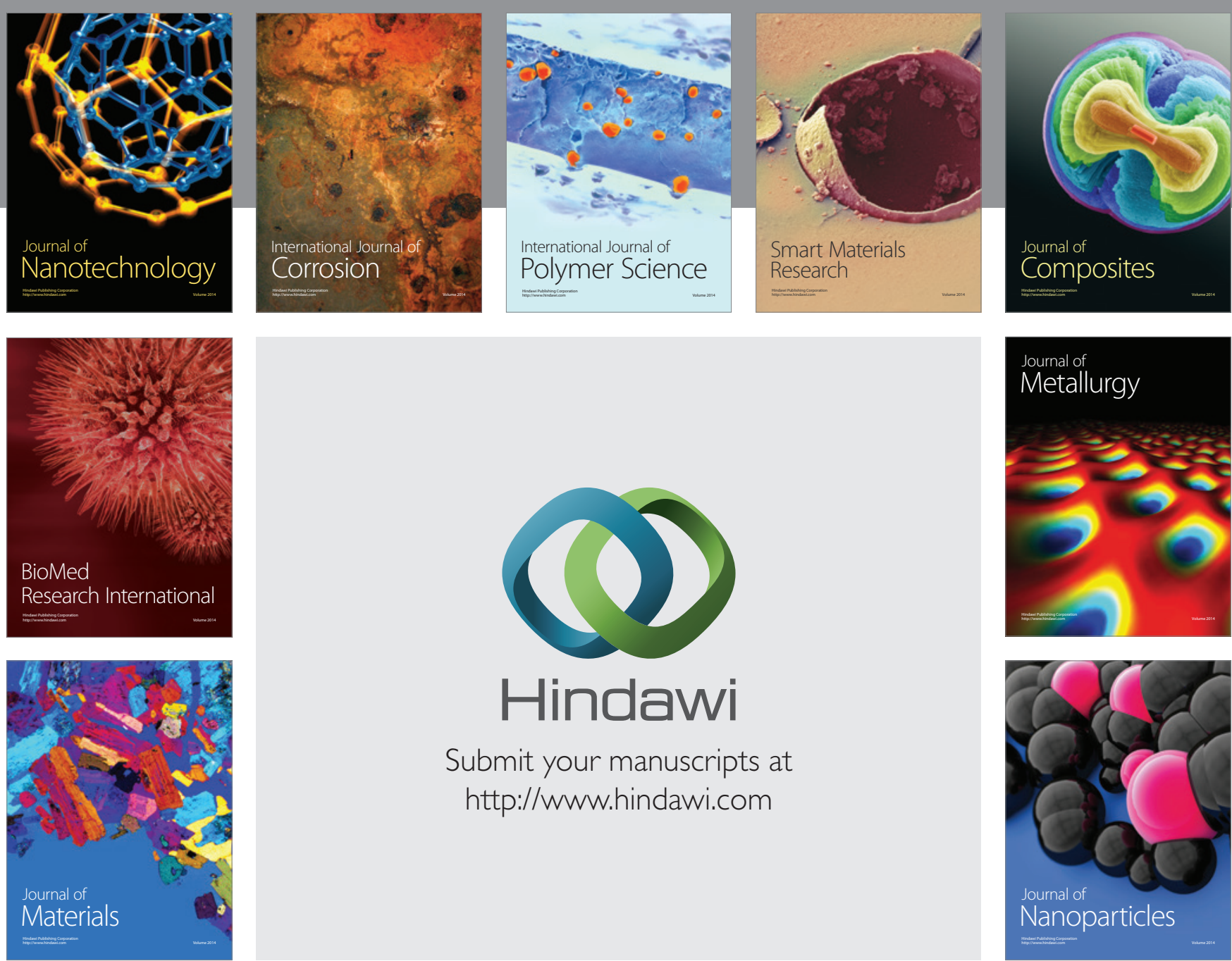

\section{Hindawi}

Submit your manuscripts at

http://www.hindawi.com

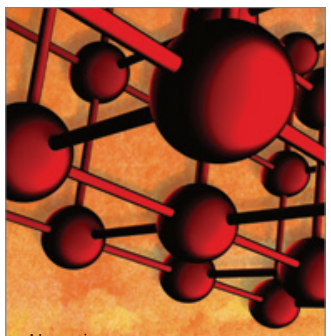

Materials Science and Engineering
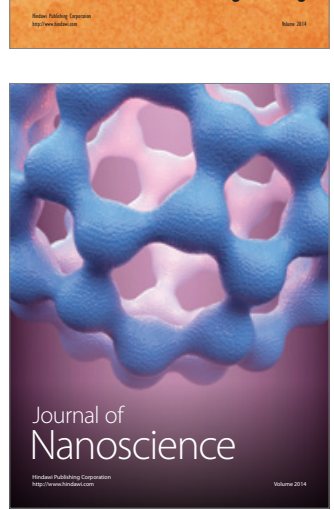
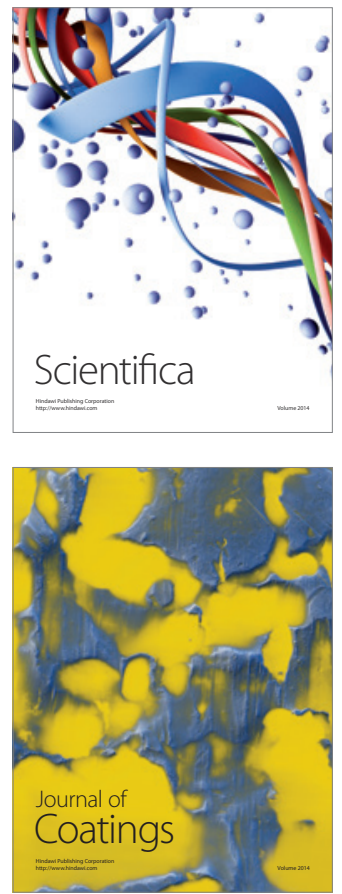
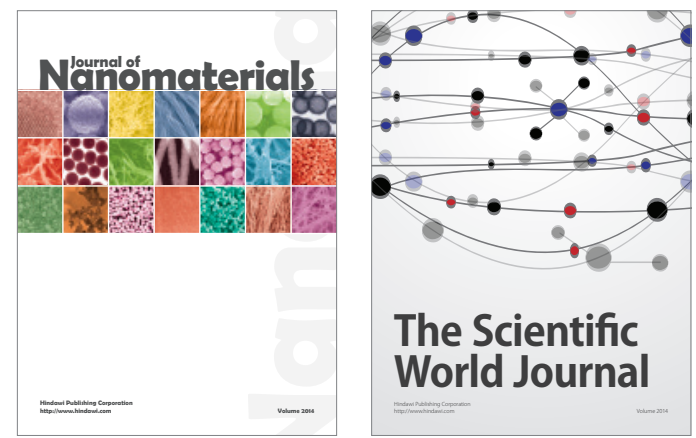

The Scientific World Journal
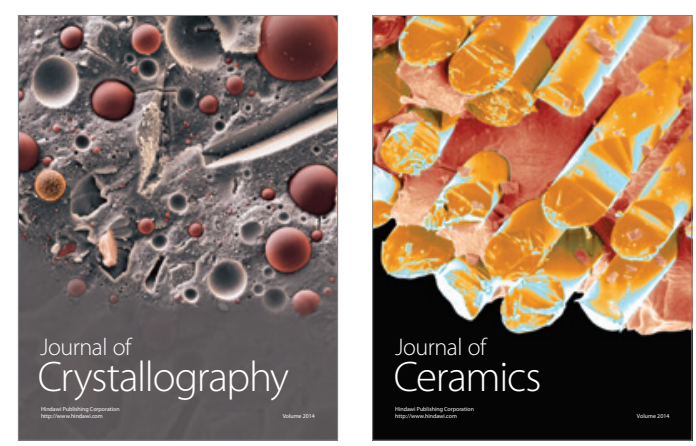
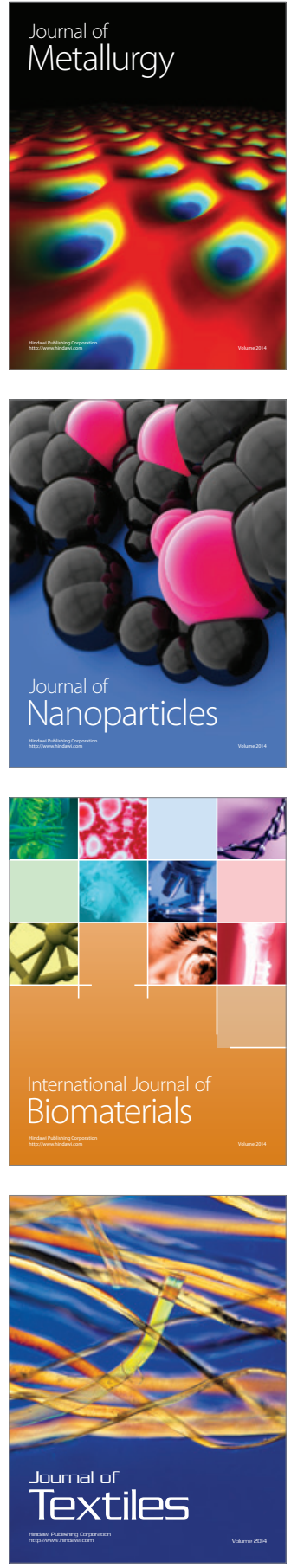